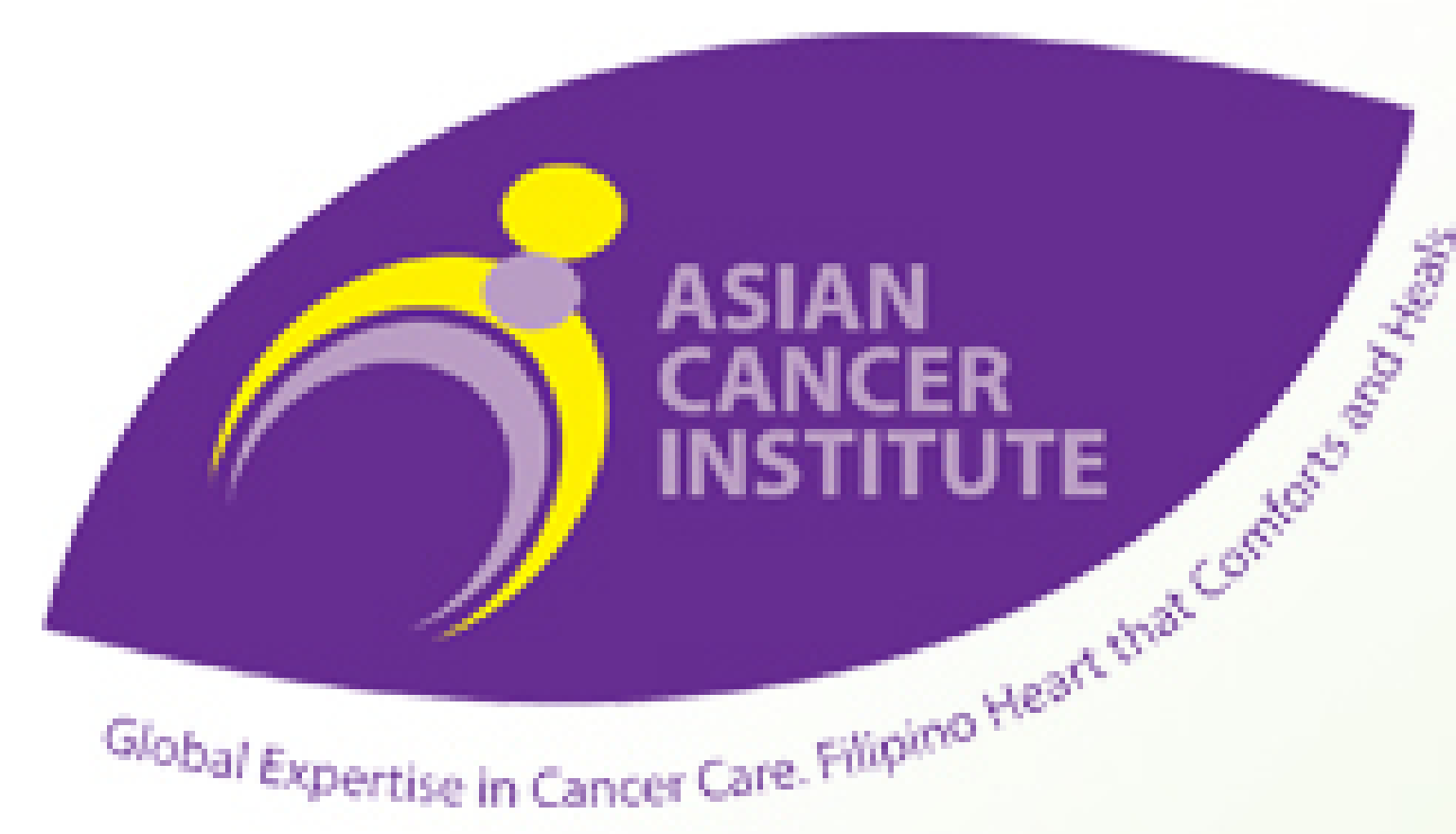

MASCC/ISOO Annual Meeting 2018, Abstract Number: MSCC8-0330

\title{
ELECTROCHEMOTHERAPY FOR THE PALLIATIVE TREATMENT OF CUTANEOUS METASTASIS AND MALIGNANT WOUNDS AMONG FILIPINO PATIENTS
}

\author{
Claire Marie Reyes-Habito', Mary Ondinee Igot', Eunice Theres Cutillar', Corazon Ngelangel'1 \\ 'Asian Hospital and Medical Center, Alabang, Muntinlupa City, Philippines
}

\section{INTRODUCTION}

Cutaneous metastasis and malignant wounds decrease the quality of life of cancer patients. Electrochemotherapy is a localized treatment which enhances the uptake of chemotherapy through the application of electric pulses. This observation study was done to evaluate the efficacy, safety, and clinical outcome of a four-electrode electrochemotherapy device (IQ Wave ${ }^{\circ}$ ) for the treatment of cutaneous metastasis among Filipino patients.

\section{METHODS}

Thirteen cutaneous metastasis were treated with 1-5 electrochemotherapy sessions at least 4 weeks apart from February to November 2017. Bleomycin or Cisplatin was given intratumorally, intravenously, or both based on the ESOPE protocol, prior to the application of electric pulses under sedation. Concomitant chemotherapy was given in 9 patients while 4 had none. Lesions were measured using a ruler and patients were evaluated using the RECIST criteria every 4 weeks.

\section{RESULTS}

There were 9 BRCA patients, 1 Head and Neck SCCA patient, and 1 sarcoma patient. Small BRCA cutaneous metastasis lesions objectively resolved in 2/13 patients after two sessions. Large fungating tumors in Head and Neck CA and Sarcoma and two large ulcerating BRCA tumors were palliatively controlled, with decrease tumor exudate, bleeding, and pain after 1 session. Disease progression, however occurred within 4 weeks post electrochemotherapy in $8 / 13$ patients. Adverse events were: post procedure pigmentation (12), itchiness (7), post-procedure pain (3), skin infection (2), skin ulceration (1), nausea and vomiting (1), rash (1).

\section{CONCLUSION}

Electrochemotherapy can be used for the palliative control of cutaneous metastasis/ malignant wounds, with minimal side effects.

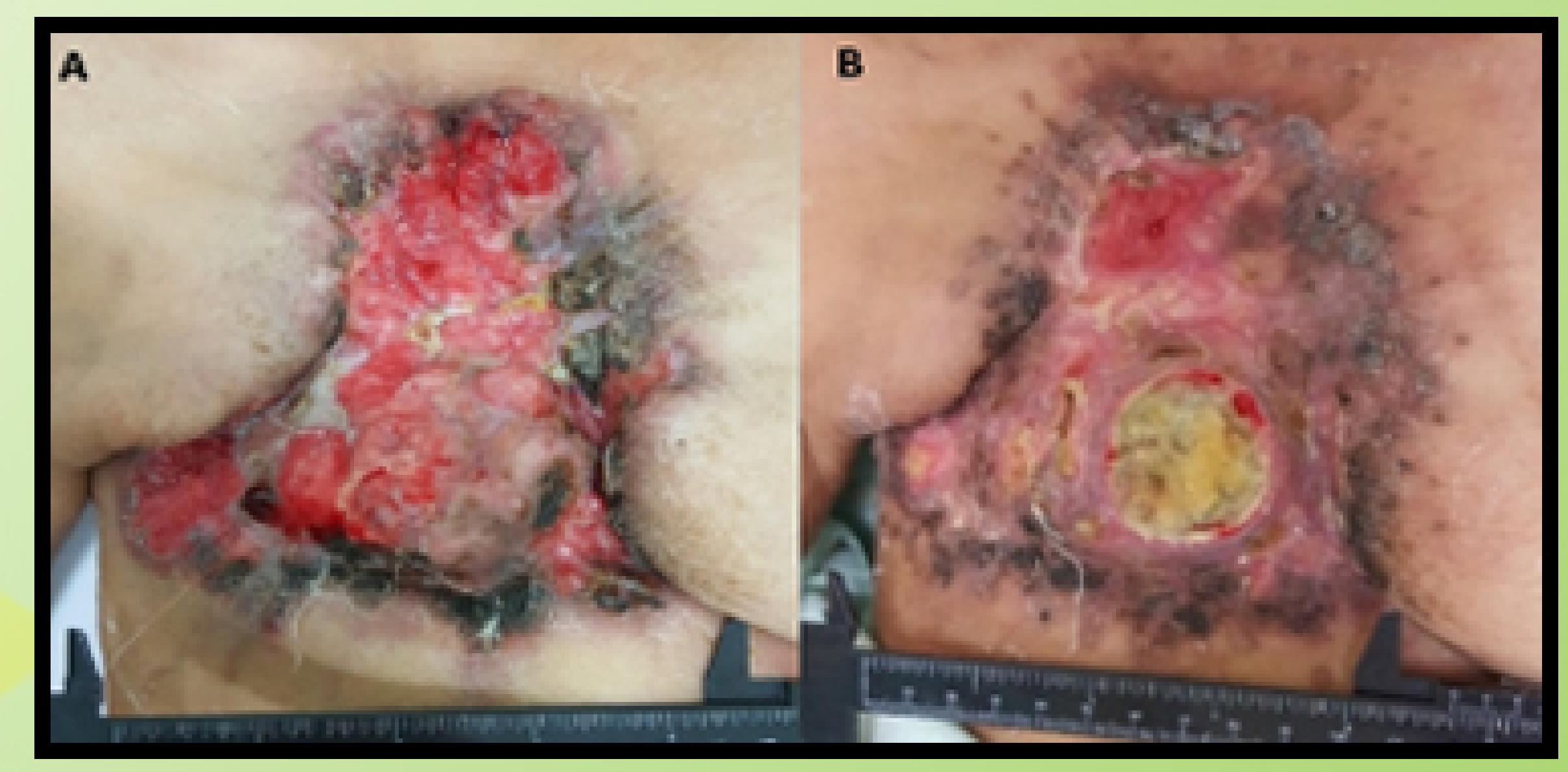

Figure 1. Breast Cancer patient before $(A)$ and 5 weeks after electrochemotherapy $(B)$

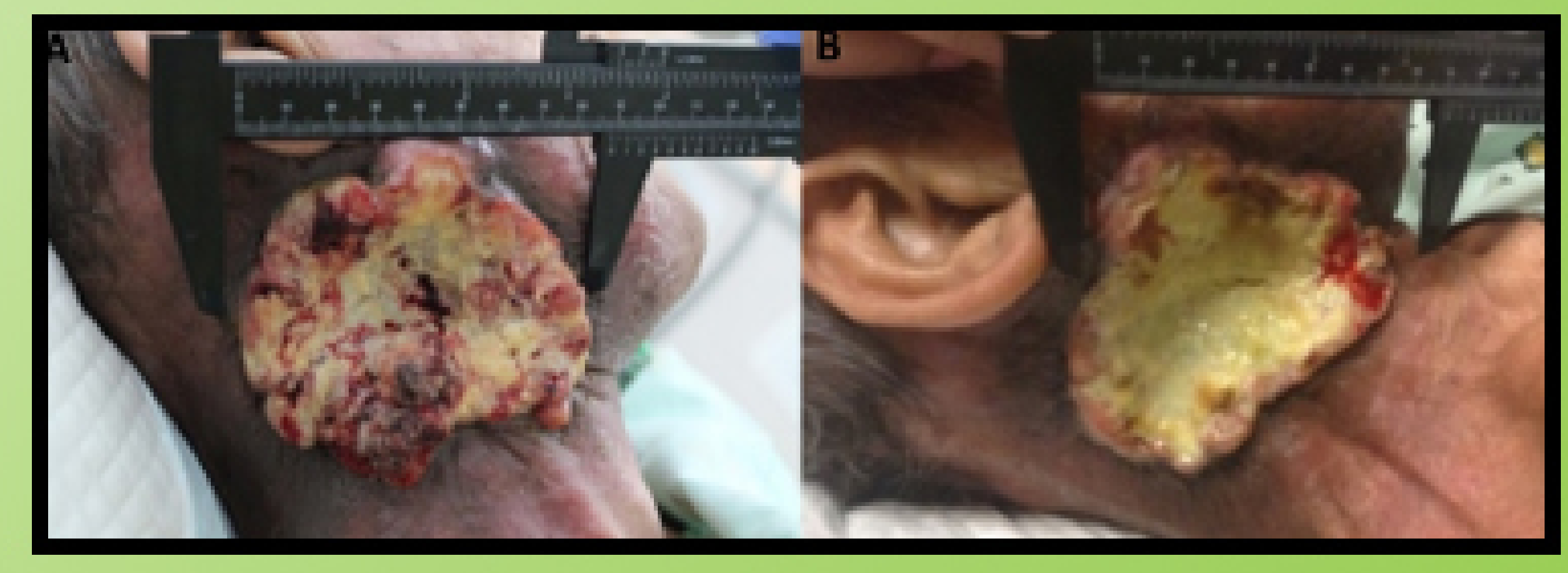

Figure 2. Head and Neck SCCA patient before (A) and 4 weeks after electrochemotherapy (B)

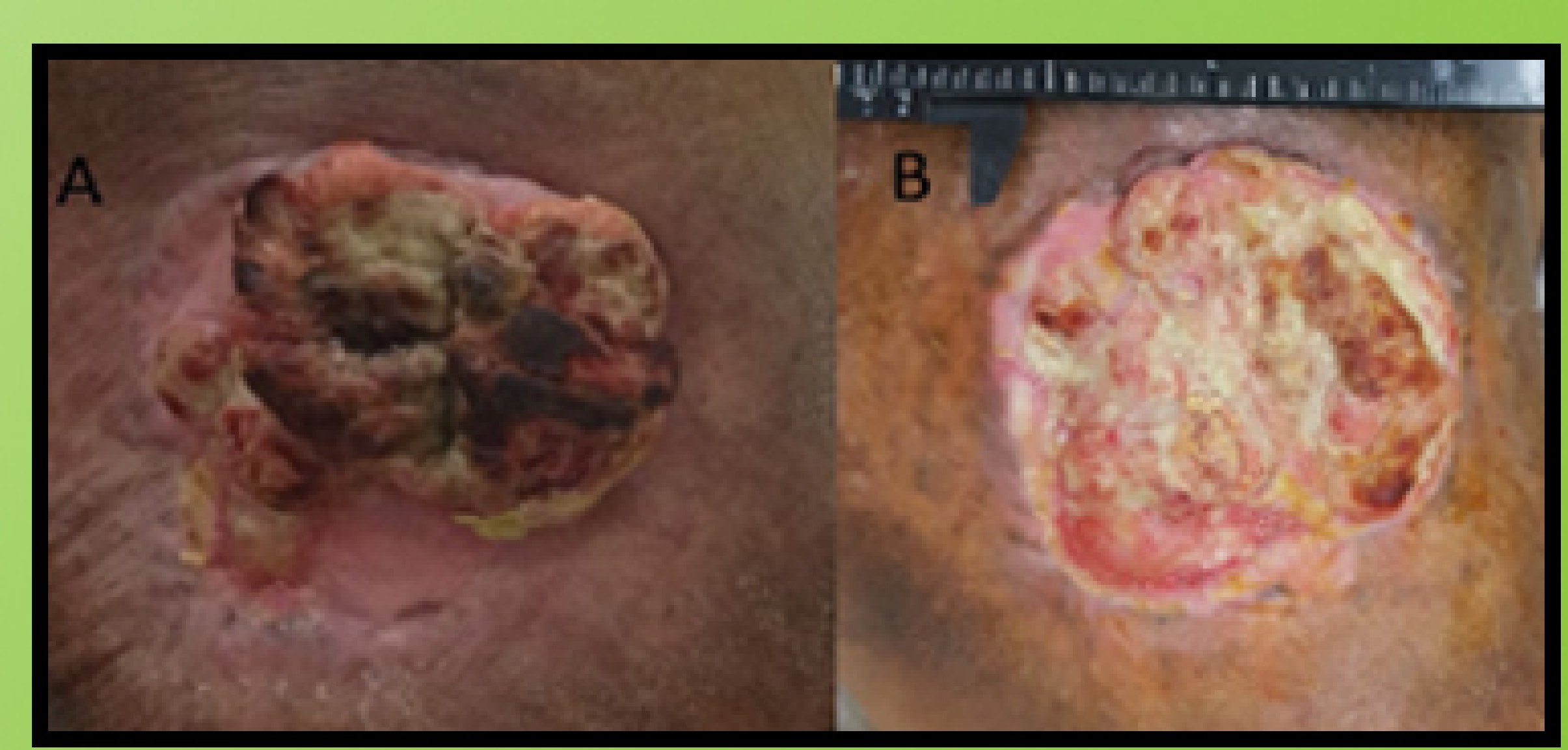

Figure 3. Sarcoma patient before (A) and 4 weeks after electrochemotherapy (B) 